\title{
Teletactile system based on mechanical properties estimation
}

\author{
Mauro M. Sette*, Hendrik Van Brussel and Jos Vander Sloten \\ Department of Mechanical Engineering, Katholieke Universiteit Leuven, Leuven, Belgium
}

\begin{abstract}
Tactile feedback is a major missing feature in minimally invasive procedures; it is an essential means of diagnosis and orientation during surgical procedures. Previous works have presented a remote palpation feedback system based on the coupling between a pressure sensor and a general haptic interface. Here a new approach is presented based on the direct estimation of the tissue mechanical properties and finally their presentation to the operator by means of a haptic interface. The approach presents different technical difficulties and some solutions are proposed: the implementation of a fast Young's modulus estimation algorithm, the implementation of a real time finite element model, and finally the implementation of a stiffness estimation approach in order to guarantee the system's stability. The work is concluded with an experimental evaluation of the whole system.
\end{abstract}

Keywords: Tactile feedback, haptic, virtual environment, Kalman filter

\section{Tactile feedback in surgery}

In common clinical and surgical practice, tactile feedback is considered as a real diagnostic tool. Many diseases cause hardening of organ tissues, allowing the doctor to identify them using only the sense of touch. Commonly the tactile sense is used for locating tumours, identifying gallstones as a harder ball embedded in a soft background, or localizing a vessel's path, which is identified as pulsing bodies. In the last years many surgical procedures have been converted from open to minimally invasive. Open surgery is performed by accessing internal organs via a wide incision in the patient's skin. These wide openings allow the doctor to reach anatomical structures with his hands and thus perform accurate palpation inspections. During min-

*Corresponding author. E-mail: Mauro.Sette@ mech.kuleuven.be. imally invasive procedures the access to the body is achieved with small incisions in the patient's skin. The surgeon inserts long-shaft surgical instruments through these openings, while the sight is guaranteed by a laparoscope. If on one side minimally invasive surgery allows trauma reduction with subsequent reduction of pain and recovery time after the surgery, on the other the sense of touch is strongly reduced when this last technique is used. Tactile feedback is even completely absent when the direct manipulation of a minimally invasive instrument is substituted with telemanipulation. A telemanipulation system is a device that enables a person to perform manual operations while separated from the site of work (an example of a commercial system is the da Vinci Robotic Surgical System (Intuitive Surgical, Mountain View, CA, USA)). It is then extremely important to restore the tactile sense in the telemanipulation procedures, developing a device that 
enables a person to touch an object or surface while separated from the site where the object/surface is, using the analogy with the telemanipulation system it can be called teletactile system.

\section{A teletactile feedback system for minimally invasive surgery}

This work presents the study of a new system for the restoration of tactile feedback in minimally invasive surgery. The tactile sense is a complex sense: it provides information about shapes, stiffness, and texture. This is the reason why the design of a complete tactile feedback system is not an easy task [31]. For this reason we decided to limit the field of application of the system to only a single tactile tasks: the localization of tumours in an organ. As an example, we will refer to the procedure of liver metastasectomy, that is, surgical removal of metastases (tumours) from the liver. The search for metastases is seen as a procedure of looking for a harder ball embedded in a soft background, and this is the task that will be analysed during the system development.

Nicholls and Lee [15] define a tactile sensor as a device or system that can measure a given property of an object or contact event through physical contact between the sensor and the object. Such a tactile sensor is based on the interaction force between an object and the sensor. If we transfer this definition to the case of searching for liver metastases, we can make a mental scheme in which we have a certain volume with non-homogeneous structure and we measure the distribution of reaction forces observable on the surface when the volume is subjected to a compression. From a certain point of view, we measure not the real material stiffness but only how this stiffness distribution affects the reaction force. This measurement gives information on the surface stiffness resulting from the interaction of different deep layers with different stiffnesses. It could be useful to have the information on the stiffness distribution in the whole volume because it can happen that important information has a too weak effect on the surface stiffness, and identifying the hard inclusion becomes difficult. This is the idea driving this work: imagine a system where, instead of the surface reaction force, we could measure the stiffness distribution directly by means of a quantitative elastography approach [29]. What would be the advantage of this approach? How we could feedback the tactile information to the user? What would be the technical problems in terms of reliability? Would this system be fast enough to create a realistic feeling?

This work is going to propose an implementation of such a teletactile system, and is going to analyze its properties and performances.

\section{System design and implementation}

At a concept level a teletactile system could be designed as a tool for a robotic system, (e.g. the da

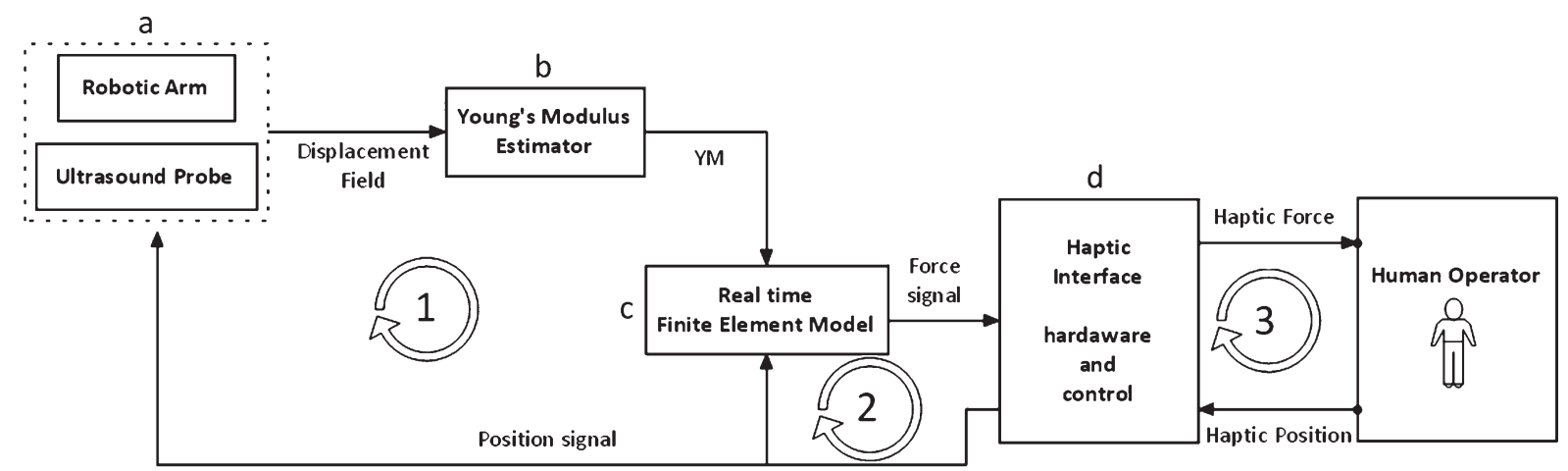

Fig. 1. The teletactile system is composed of four different modules. a) Module that controls the position of the "Ultrasound probe" connected to the "Robotic arm". b) Module for the estimation of mechanical properties, the "Young's modulus estimator. c) Module to synthetize the tactile input for the tactile display with the implementation of a semi-virtual environment, the "Real time finite element environment". d) Module for the control of the "Haptic interface". The for modules are integrated on three parallel loops. Loop (1) is the image acquisition system (robotic arm and ultrasound system) and ultrasound image processing unit for the estimation of the Young modulus data; loop (2) the Young modulus is integrated into a finite element (FE) model interfaced with a haptic device. Loop (3) represents the interaction between the human operator and the haptic interface. 
Vinci System) composed of four different modules running in three different loops, as shown in Fig. 1:

a - A module that controls the position of the "Ultrasound probe" connected to the "Robotic arm";

$\mathrm{b}$ - a module, for the estimation of mechanical properties, the "Young's modulus estimator";

c - a module, to synthetize the tactile input for the tactile display with the implementation of a semi-virtual environment, the "Real time finite element environment"; and

$\mathrm{d}$ - a module implementing the "Haptic interface".

The following sections are going to describe in details the four blocks composing the system.

\subsection{Module a-Control of the ultrasound probe position through the robotic arm}

A laparoscopic ultrasound probe that is manipulated by a robotic arm, for example the da Vinci CANVAS described in [14], composes the hardware part of this block. Figure 2 describes the adaptation of a laparoscopic ultrasound probe as the tool for the da Vinci Surgical Robot System. The ultrasound laparoscopic probe described in the system is the Aloka End-fire Laparoscopic probe (Aloka, Mitaka-shi, Japan), frequency range 3 - $8 \mathrm{MHz}$, diameter of the insertion part $12 \mathrm{~mm}$, and length of the insertion part $375 \mathrm{~mm}$. This module controls the position of the ultrasound probe in order to acquire images of the area under investigation. The position of the ultrasound probe is controlled by the position of the surgeon's "finger" i.e. the location in which the surgeon wants to touch. Anticipating the description of the other blocks can be observed that, the ultrasound image is acquired an processed in the loop 1 (Fig. 1), the will keep running only in the loop 2 until the surgeon finger's goes out of the acquired area, in this case a new segment should be acquired and processed. Here follows two possible solution on how to optimize the coupling between the finger and the ultrasound probe:

(1) While the system is engaged in the loop 2 at the time $\mathrm{T}$, calculate an adjacent portion of volume that will be explored at the time period $\mathrm{T}+1$ (Fig. 3).

(2) Calculate a second slice along the volume's transverse direction (Fig. 4). In this case, the model for the virtual environment will be updated slice by slice. This solution implies the use of a three-dimensional model. The main advantage is the possibility of creating the input for a bi-dimensional matrix tactile display. For this case, the requirement for the $f_{u}$ is difficult to define because it depends strictly on the haptic device used and on its resolution. However, we can only guess that it could be higher that the one described before.

Both solutions could be implemented efficiently. Each solution is strictly dependent on the haptic interface used. For a kinaesthetic feedback device, the first solution would be preferable because in this case it is necessary to explore the surface along one direction. The second may be more suitable when the system feeds back the information through a tactile device and bi-dimensional information is needed. In this work, the application for a kinaesthetic device will be developed, and thus a bi-dimensional model will be implemented, allowing exploration along only one direction.

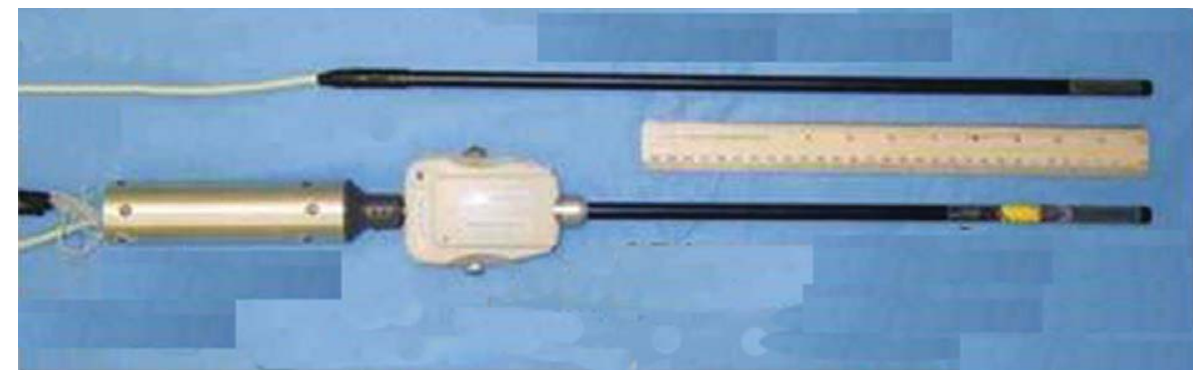

Fig. 2. The picture represents a normal Aloka (Aloka, Tokio, Japan) ultrasound probe for laparoscopic procedures (above), and the integration of the probe in the da Vinci Surgical System (Intuitive Surgical, Mountain View, CA, USA). This integration was performed by Leven and it is described in [14]. The Aloka probe has a diameter of $12 \mathrm{~mm}$ and a length of $375 \mathrm{~mm}$. 


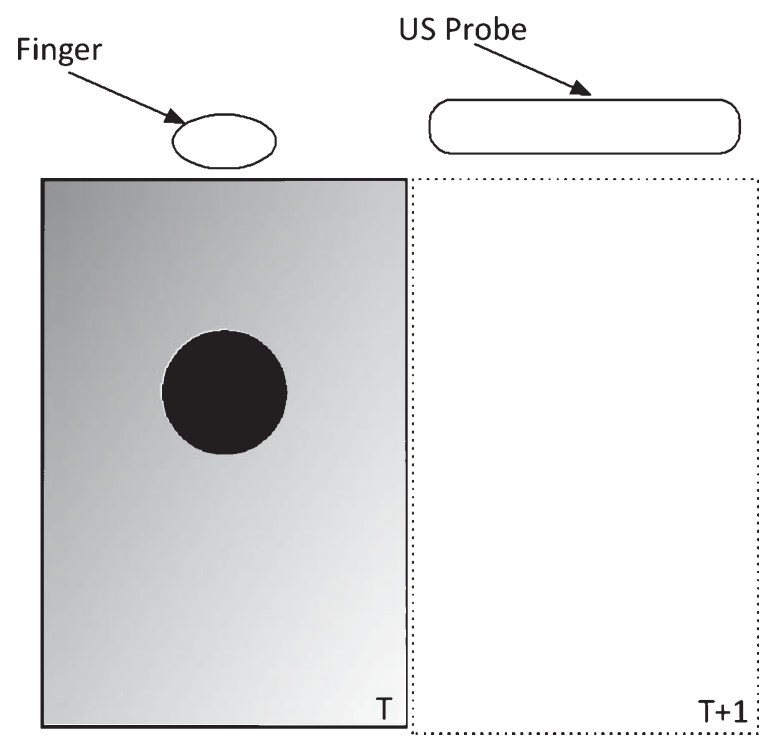

Fig. 3. Two different strategies for the exploration of the surface by the ultrasound probe have been presented. The first strategy proposes that: At the time $\mathrm{T}$ the portion explored was calculated at the time $\mathrm{T}-1$, while the system calculates the portion that will be explored in $\mathrm{T}+1$.

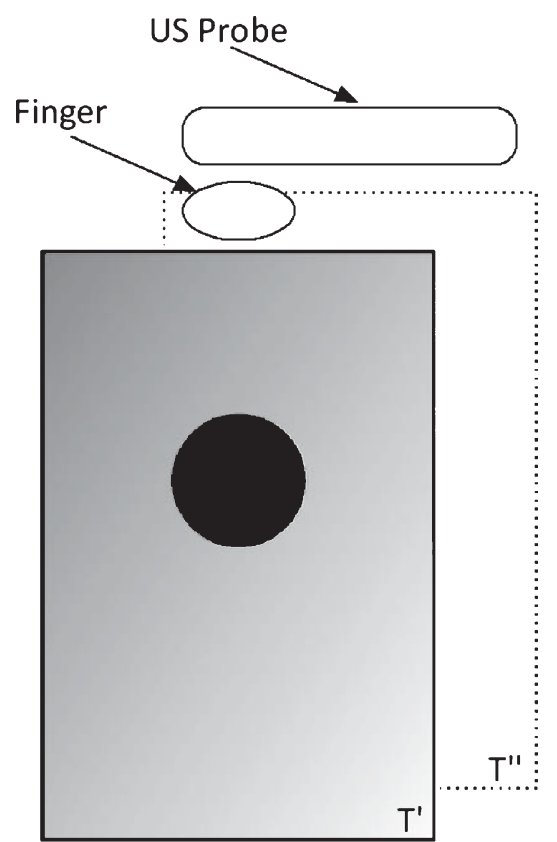

Fig. 4. Two different strategies for the exploration of the surface by the ultrasound probe have been presented. The second strategy proposes that: The different sections of a certain volume are calculated sequentially. The volume will become available slice by slice in the virtual environment.

\subsection{Module b-Young's modulus estimator}

The time requirements for the Young modulus estimation depend on exploration speed at which normally the fingers explore a surface. The block b calculates the Young modulus distribution of a finite volume portion, and as long as the finger stays in this portion, no new calculation is required. The best control algorithm that is needed to realize this synergy between loop 1 and loop 2 is still the object of investigation. If we assume that at each period $\mathrm{T}$ we are able to calculate a surface with width $w$ (which is the width of the Laparoscopic-US-probe's footprint under the condition of using a linear probe) and considering that the maximum exploration speed $v_{e x}$ for the finger is 120 $\mathrm{mm} / \mathrm{s}[24]$ the update frequency $\left(f_{u}\right)$ of loop 3 is given by:

$$
f_{u}=\frac{v_{e x}}{w}
$$

If we consider that footprint of the probe in Fig. 2 is $60, \mathrm{~mm}$, the update frequency for the loop has an upper limit of $2 \mathrm{~Hz}$. This means that all the calculations must be solved in less than $500 \mathrm{~ms}$. This represents the worst case scenario in which the finger is sweeping the surface at maximum speed. This feature influence strongly the choice of the algorithm to be used for the Young modulus estimation. The tissue stiffness (or Young modulus) estimations is performed by solving an inverse elasticity problem, which needs as input the displacement field within the tissue, calculated from a set of ultrasound images, and the boundary conditions. The output is the estimation of the examined volume's mechanical properties. This technique, applying iterative or direct methods, has been extensively studied by the authors and has already been described in previous publications. For a complete explanation of the algorithm we refer to: $[3,8,9,19-23,26-29,34]$. These previous work showed how different set of boundary conditions could be used, this system make use of a set of mixed boundary conditions, both traction (force) and displacement boundary conditions are applied to the model. The traction boundary conditions are estimated with a pressure sensor located at the US probe tip. This pressure sensor has two basic functions: (i) to acquire the information to set the boundary conditions for the solution of the inverse elasticity problem, and (ii) to integrate a force control loop for the exploration of the organ surface as described by Trejos in [35]. 
The estimation of mechanical properties can be implemented using different strategies, the state of the art lists two different approaches, iterative and direct. Both approaches have advantages and drawbacks that must be analysed in order to make the correct choice for an eventual development. Iterative methods have been studied mainly; they have the big advantage of being less sensitive to noise as well as stable; in fact they are based on the solution of a non-linear least squares problem that can be solved with optimization algorithms like the Gauss-Newton or Levenberg-Marquardt method [18]. Direct methods are faster but they are more sensitive to noise and boundary conditions approximations. However if a good level of strain estimation could be achieved this last class of method is surely promising and can be implemented efficiently on a real time device.

There exists a third possibility for the stiffness estimation, which is the use of the shear wave elastography; this technique is very promising, and eventually allows a fast estimation of the mechanical properties. However it has not been studied by the authors of this work and will not be considered in the discussion of possible systems. Nevertheless, it remains an interesting approach that needs to be further analysed. For more detail on this approach refer to Bercoff et al. [5], Sandrin et al. [32].

\subsection{Module $\mathrm{c}$-Real time finite element model}

Let's suppose that the information on the stiffness distribution is efficiently estimated from the intra operative ultrasound images, as example we report the stiffness distribution calculated with a direct method from a set of ultrasound images: Fig. 5 (picture taken from [30]). The problem that is still open is how to render this information to the operator. The system presented here is a semi-virtual system, that is, the operator interacts with a virtual environment that is

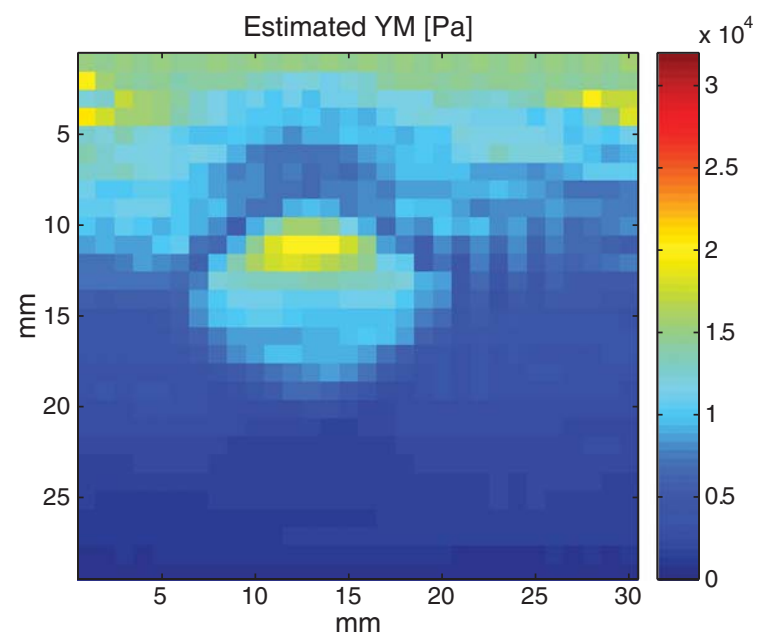

Fig. 5. The stiffness distribution is calculated with an elastography based approach. Here is reported an example of stiffness distribution estimated with a direct elastography method. From [30].

updated in real time. Thus, the architecture at its basis can be treated, at least for the human interaction part, as the architecture of a virtual reality application with haptic feedback. Salisbury provides an example of such architecture in [25], and Fig. 6 illustrates the concept. The simulation engine is responsible for computing the virtual environment's behaviour over time, the rendering blocks compute the responses for the visual, auditory, and haptic feedback, and finally between the human operator and the virtual environment there are the physical transducers. Although they present interesting and complicated challenges, the authors will not treat the visual and auditory simulations and their rendering to the surgeon. Instead, possibilities, problems, and eventual solutions for the haptic simulation and rendering will be discussed. Compared to the Salisbury scheme (Fig. 6), the scheme presented in Fig. 1 is its analogous as highlighted in Fig. 7.

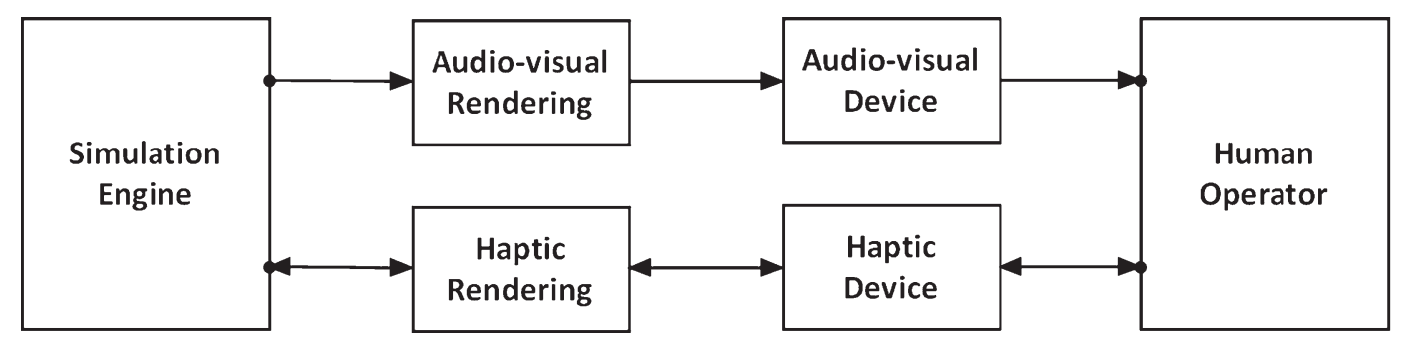

Fig. 6. A basic architecture for a virtual reality application classically includes two channels: The audio visual channel and a haptic channel. Those two channels with the relative renderings realize the connection between the simulation engine and the human operator. From Salisbury [25]. 


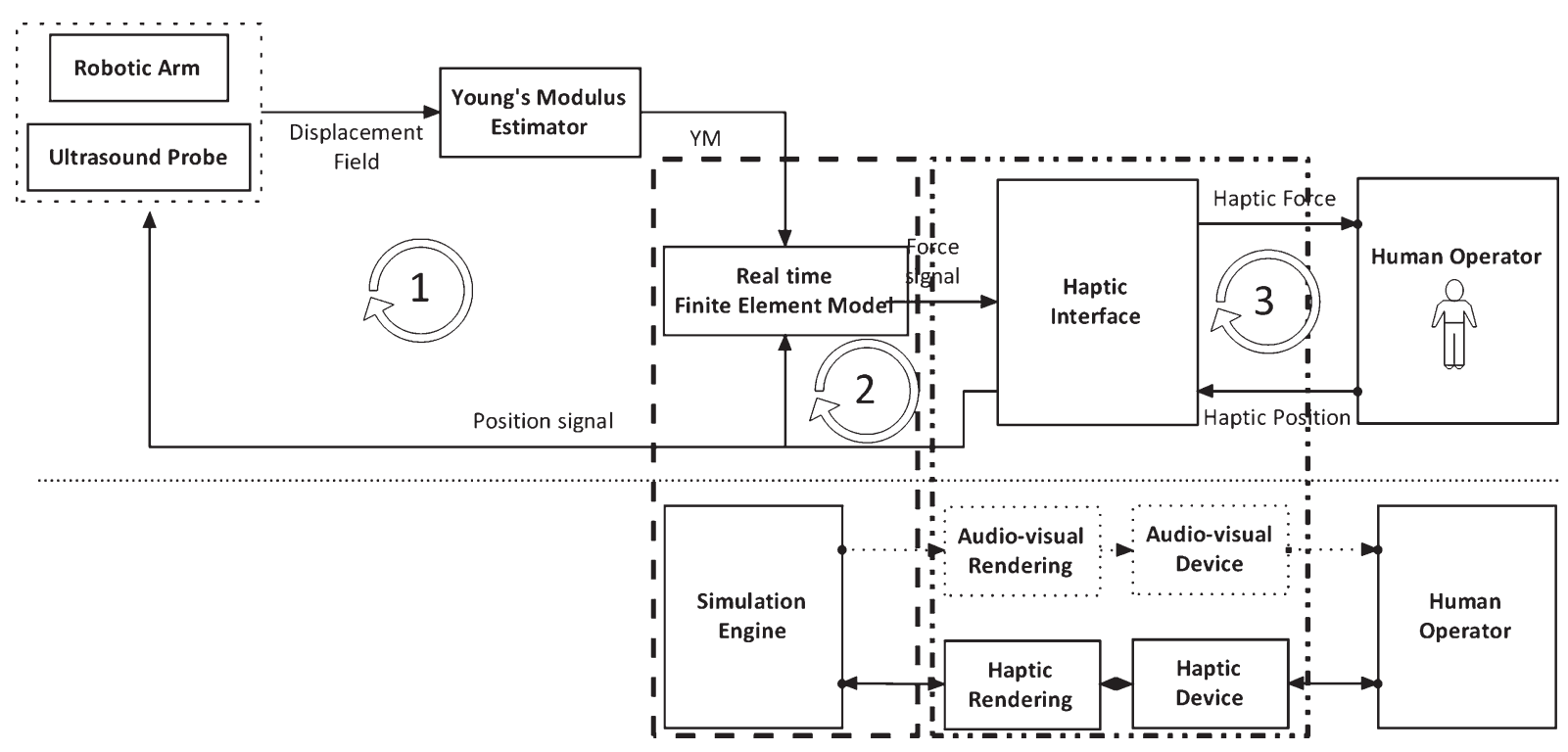

Fig. 7. Analogies between the scheme presented here and Salisbury's scheme.

The function of this block is to calculate the interaction forces between the virtual surgeon's finger exploring the volume, and the model built with the on-line estimated mechanical properties. In order to calculate such a reaction force we make use of the theory of elasticity; the equation describing the mechanics of continuum is integrated numerically using a finite element (FE) method, as explained in [1]. The FEM is built in two dimensions; the elements used are quadrilateral elements and the number of nodes should guarantee a spatial resolution equal to the finger tactile resolution, that is, $1 \mathrm{~mm}$ [15]. The contact control block calculates the deformation applied to a set of nodes included in the area of contact between the avatar and the model. This set of deformations is the displacement boundary condition applied to the model. The global equation, representing the relationship among all the FE's nodal values, is obtained by assembling element wise equations by imposing inter-element continuity of the solution and balancing for inter-element forces (detailed in [1]). This can be reduced to solve a system of differential equations:

$$
M \ddot{u}+D \dot{u}+K u=F
$$

where $u$ is a $2 n$-element, nodal displacement vector; $\dot{u}$ and $\ddot{u}$, the respective velocity and acceleration vectors; $F$, the external force vector; $M$, the mass matrix of size $(2 n, 2 n) ; D$; the damping matrix; and $K$ the stiffness matrix, while $n$ is the number of nodes in the
FEM. During surgical manipulation, dynamic effects are negligible [4], and thus it is sufficient for the quantitative elastography algorithm to calculate only the elastic properties of the examined model. The FEM was built through three different steps:

(1) The problem to solve was identified. The numerical solution which approximates the solution of the elasticity problem, or the Navier-Lamé problem [1], was found.

(2) The FEM was implemented and the boundary conditions were integrated. The numerical integration of the constitutive equation leads to the definition of a linear system. The coefficient's matrix $K$ is built considering the model composed of quadrilateral meshes and linear hat functions $(2 n, 2 n)$. The vector of the unknowns $x(2 n)$ is the vector of the nodal displacements. Finally the vector of the external forces $f(2 n)$ is applied on each node ( $n$ is the number of nodes). The multiplication factor 2 is the number of degrees of freedom (d.o.f.). The formal description of how to build the FEM is given in [1]. For simplicity we will assume it is possible to build the FEM in the form:

$$
K x=f
$$

The Dirichlet boundary conditions (DBC), which are the displacement imposed at the 
nodes, will be applied by means of the Lagrange multipliers. The displacement is fixed in one specified direction and is possibly free in others. This can be translated in defining the system:

$$
B U=w
$$

where the matrix $\mathrm{B}$ has dimensions $(d, 2 n)$, where $d$ is the amount of d.o.f. that need to be constrained with the DBC and $x_{d}$ is the value assigned to the degrees of freedom on $\Gamma_{D}$. The matrix $B$ is initialized to values of zero, and for each row $d$ the $2 n$-elements are set to one. Finally, $w$ contains the array of $x_{d}$ values. Coupling this set of conditions (4) through Lagrange parameters with (3) leads to the extended system:

$$
\left(\begin{array}{cc}
K & B^{T} \\
B & 0
\end{array}\right)\left(\begin{array}{l}
x \\
\lambda
\end{array}\right)=\left(\begin{array}{l}
f \\
w
\end{array}\right)
$$

(3) The linear system was solved with a classic algorithm like Gaussian elimination [2]. The Lagrange multipliers approach is more convenient when it is necessary to implement the FEMs in a real time environment. This allows the calculation of the reaction forces on the Dirichlet nodes with a small computational load, and provides a fast and reliable system to be implemented in real time [4]. In practice the displacements induced by the contact with the avatar are assigned to the vector $x_{d}$ and applied to the d.o.f. d. This technique makes the algorithm faster because it avoids the stiffness matrix's re-computation at each loop.

\subsection{Module d-Haptic interface, hardware and control}

The haptic interface (Fig. 7) block includes the control algorithm for the haptic interface, a feature of fundamental importance because it covers the gap between the simulation's updating frequency (lower) and the haptic control's required updating frequency (higher) and contact control (the block that identifies the interaction between the haptic interface and the model).

\subsubsection{Control of the haptic feedback}

In past years researchers have been working on finding a solution able to give a realistic feeling and at the same maintaining the stability of the controlled system. The aim of this section is not to give an exhaustive explanation of the complex theory behind the haptic feedback and telemanipulation, but instead to treat one of the main problems that could be present when interacting with a virtual (or semi-virtual) haptic environment: the instability generated by the low update rate in the simulation engine. The virtual environment updating frequency is dependent on the implementation of the FEM and the type and size of FEM used. Colgate et al. $[6,7]$ have demonstrated how the update frequency can affect the system stability. With small changes, Colgate's work can be adapted for the presented application as illustrated in Fig. 8. The simulation engine works in a discrete time domain; it calculates the reaction force $F E M$ in response to a displacement $x$. The Zero Order Holder block represents the link between the discrete time domain and the

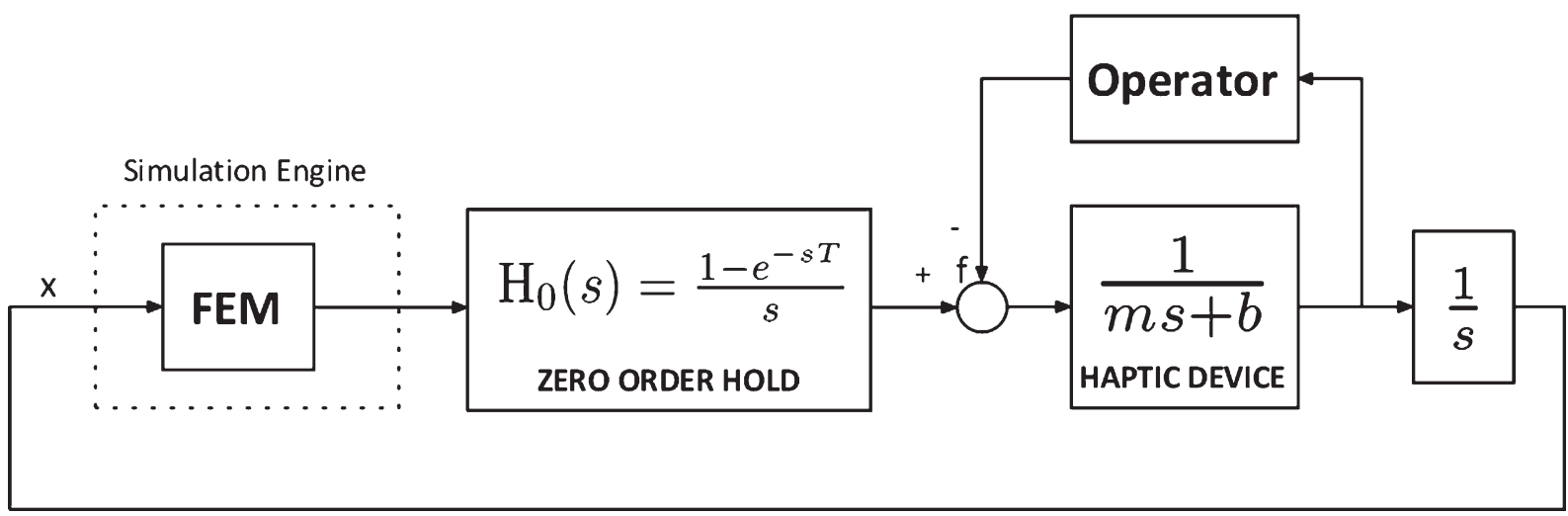

Fig. 8. System's scheme. The simulation engine integrates the solver for the finite element model (FEM). The interaction between the operator and the haptic device is modeled. Finally the link between the simulation engine discrete time domain and the real continuous time domain is modeled with a zero order hold. 
continuous time domain. Colgate's major finding is a formulation of a general rule, based on the passivity theory [6], in which the relation between the environment $H\left(e^{-j \omega T}\right)$ (in this case the FEM), the inherent display's damping $b$, and the sampling period $T$ is:

$$
\begin{array}{r}
b>\frac{T}{2} \frac{1}{1-\cos \omega T} \operatorname{Re}\left\{\left(1-e^{-j \omega T}\right) H\left(e^{-j \omega T}\right)\right\} \\
0 \leq \omega \leq \omega_{N}
\end{array}
$$

where $\omega_{N}=\pi / T$ is the Nyquist frequency.

From Colgate's rule it can be concluded that, in order to maintain stability, the parameter $T$ should be reduced. A common approach is to linearly interpolate between two values instead of holding the value of $f_{F E M}$ between two periods. A better approach could be to use a stiffness estimation scheme. This scheme was implemented using the formalism of the extended Kalman filter as previously presented by De Gersem in [10]. The Kalman estimator is based on recursive least squares and uses measurements and process noise to weigh the updates, which takes the measurement noise into account.

\subsubsection{A new concept for contact control}

The avatar, a software representation of the device's tip, represents the haptic device in the virtual environment; usually the model to be touched and the avatar are incompenetrable; that is, they can only deform, and this property reflects the real object's behaviour. In the experimental phase, this constraint will be removed, giving the user the possibility of touching under the surface; that is, touching a series of virtual surfaces generated below the surface representing the natural organ's surface. The expected advantage is the augmentation of the tactile sense, leading to a more powerful tool for the identification of metastases. In order to implement the capability of touching under the surface it is necessary to formulate a novel scheme for the interaction between the avatar and the virtual surfaces inside the volume (Fig. 9); the contact control module works for this scope. A series of surfaces internal to the model are set and the operator is free to touch along those surfaces.

\section{Materials and methods}

The first section of this paragraph explains which platform has been used for the physical implementation of the system. Then the system has been tested with respect to several aspects. The first experiment sets are aimed at characterizing the system under the performance aspects. The speed of calculation of the virtual environment has been tested, varying the model's size; the stiffness estimation has been tested against the normal stiffness; and finally the contact control strategy has been tested evidencing how different the explored stiffness appears when the boundary conditions are changed. The experimental session concludes with a series of experiments evaluating the system's performances using psychophysical tests.

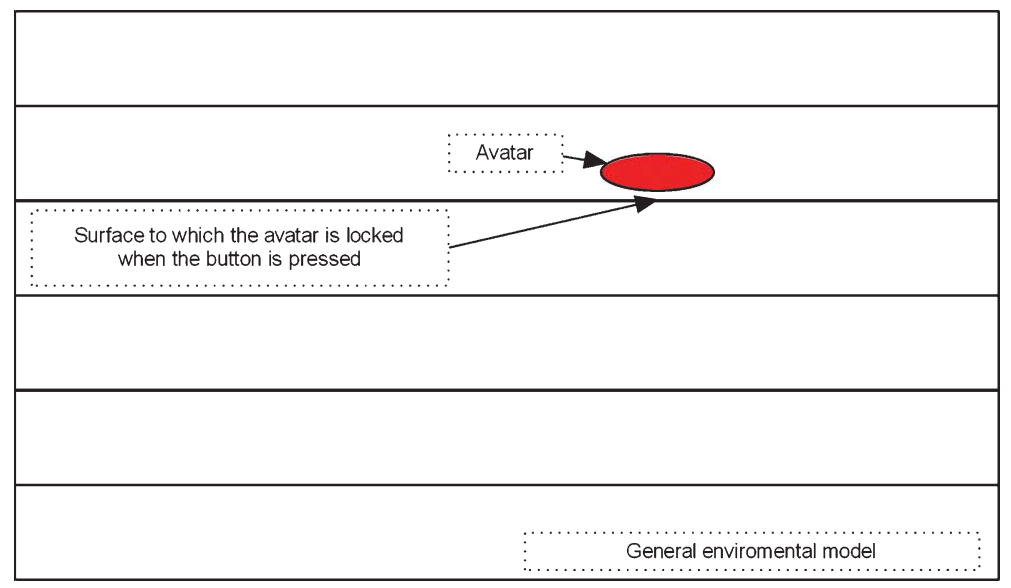

Fig. 9. Estimating the stiffness distribution within a volume, the surgeon has the possibility of touching under the natural surface. In the system's implementation the surgeon's finger is represented by an avatar. The touching surface is set by a secondary control at different levels of the virtual model. 


\subsection{Hardware and software platform}

The haptic rendering block has been implemented in the PXI Real Time System (CPU-1) (National Instruments, Austin, TX, USA) with a dedicated program written in Labview (National Instruments, Austin, TX, USA). The simulation engine block implements the FEM for the elastic interaction between bodies and was implemented using a pseudo-code software: Octave [12]. The FEM was calculated by a second processing unit (CPU-2) in order to increase the computational power available and was run on a Linux operating system (Ubuntu) (http://wiki.ubuntu.com/). The data are exchanged between the two operating systems using the TCP/IP protocol [33] and between the CPU-1 and the haptic device using dedicated digital ports. A general scheme is reported in Fig. 10. The system testing used the Phantom Premium (www.sensable.com) as the haptic interface; this device is commonly used for haptic systems and represents one of the most used haptic masters [25] (Fig. 11).

\subsection{System's performances}

\subsubsection{Effect of the Kalman filter}

As explained in Sec. 3.4.1. the stability of the system is improved by the introduction of a stiffness estimation scheme. This block uses a Kalman filter to decouple the virtual environment from the haptic interface controller. It is necessary to measure which is the difference between the stiffness estimated with the Kalman filter and the stiffness estimated in a ideal case. We expect that the Kalman filter introduces some phase lag in the estimation due to the filtering process. In order to estimate the phase lag we compared a "real stiffness" that is the stiffness used as input to the filter with the stiffness estimated with the Kalman filter. The experiment is conducted, recording the stiffness estimated during the surface exploration, the exploration was simulated shifting the node on which the force was applied from one side of the model and back at the speed of $120 \mathrm{~mm} / \mathrm{s}$, and at the same time the degrees of freedom on which the indentation displacement is applied are recorded as a function of the time. Those last data are then used to calculate the real stiffness off-line.

\subsubsection{Calculation speed over the distributed system}

A major limiting factor for the system's performance is the FE calculation time and the speed of communication between different systems. A test of the time required for the FE calculation and the communication time required to transmit the data is performed. Different FE models where tested with degrees of freedom ranging from 408 to 1071 (the FEM number of columns was kept fixed to 50 while the amount of lines was variated from 8 to 20).

\subsubsection{New concepts for contact control and stiffness augmentation}

The user perceived stiffness is measured as the ratio between the indentation force and the nodal displacement on which the force is applied. This quantity is

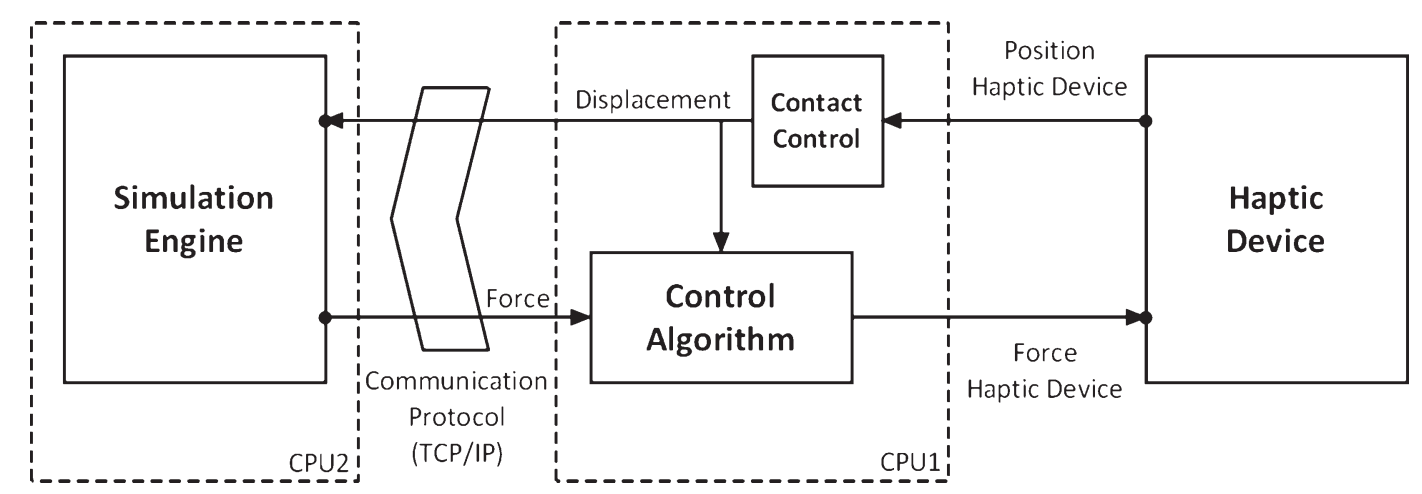

Fig. 10. The virtual environment is divided in two section each working on an independent processing unit (CPU), in the first block in calculated the interaction point of the haptic avatar with the virtual environment and there is block for the control of the haptic device; while the on the second processing unit are solved the calculation of the virtual environment. The two blocks are physically on two different units and are connected with a TCP/IP protocol. 


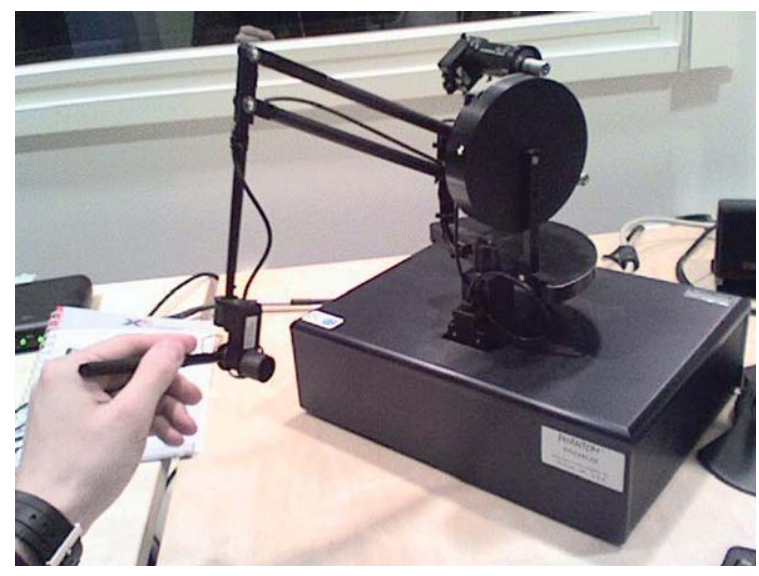

Fig. 11. Phantom haptic master (Sensable, Wilmington, MA). This mater is able of providing up to six d.o.f. force feedback.

considered as index of the stiffness perceived by the subject. A set of experiments was conducted with the aim of measuring this quantity over different touching surfaces of a FE model. Aim of this experiments is to highlight the advantages brought by the semi-virtual environment. A linear elastic FE model was created, the plain strain approximation was implemented. The mesh was built with 50 by 10 quadratic elements. In the FE model was simulated a hard inclusion embedded in a softer background. The YM of the inclusion was five times harder than the background. The model is presented in Fig. 12. Nine different touching levels were set. The first was at the "natural surface" ( $\mathrm{Y}=0 \mathrm{~m})$ and the last was at the line immediately above the hard inclusion $(\mathrm{Y}=-0.08 \mathrm{~m})$. For numerical reasons was not possible to test the surface at $\mathrm{Y}=-0.09 \mathrm{~m}$. The subject was asked to explore the surface with the haptic interface and the stiffness calculated as the ration between the applied force and the nodal displacement was recorded.

\subsection{Psychophysics experiments, subjects and protocol}

The aim of the psychophysical experiments was the testing of the system in terms of the subject's ability to discriminate a hard inclusion embedded in a soft envi-

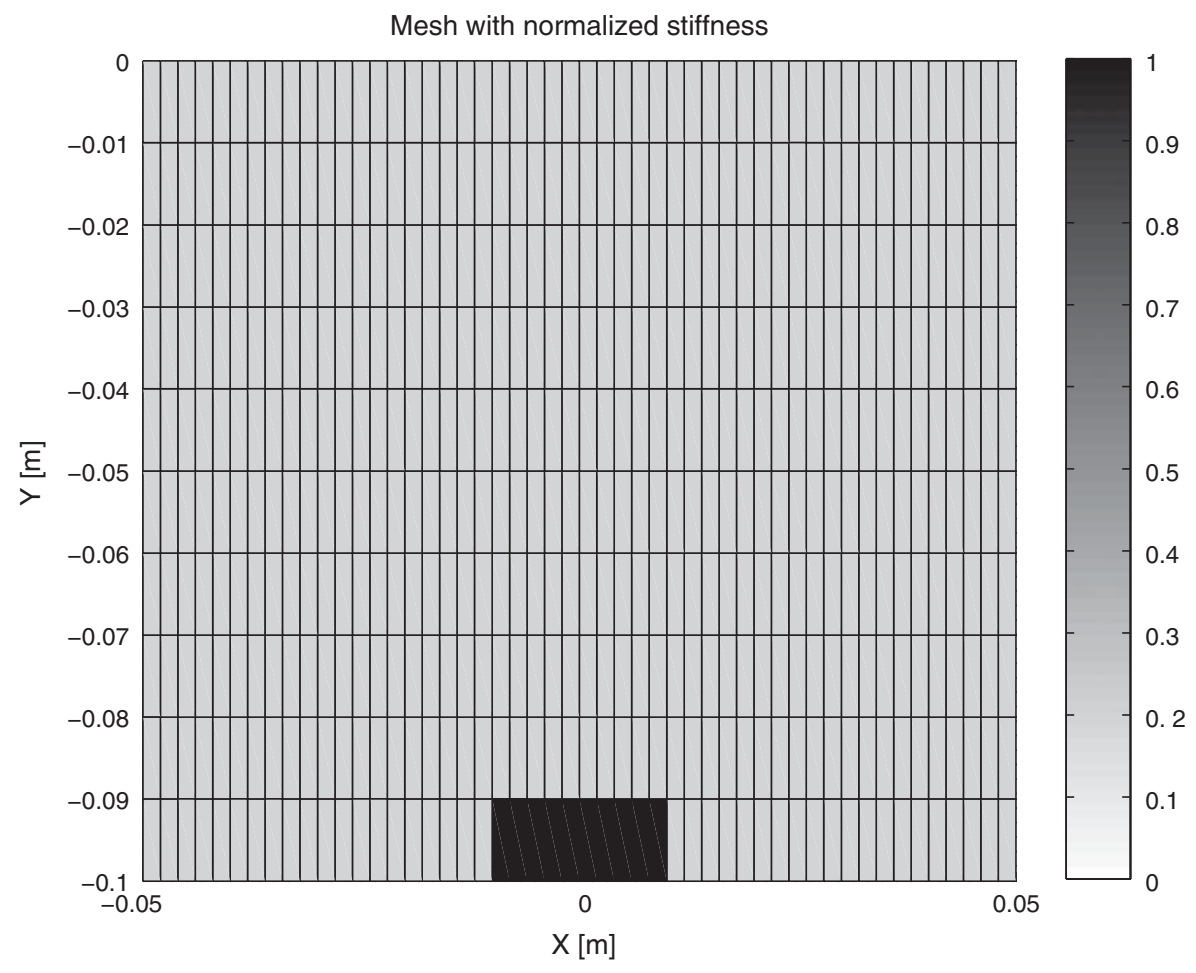

Fig. 12. The FE model used for the experiments was a linear elastic FE model, the plain strain approximation was implemented. The mesh was built with 50 by 10 quadratic elements, with dimension $10 \mathrm{~cm}$ by $10 \mathrm{~cm}$. The harder inclusion, the black spot on the bottom, was five times harder than the background. 


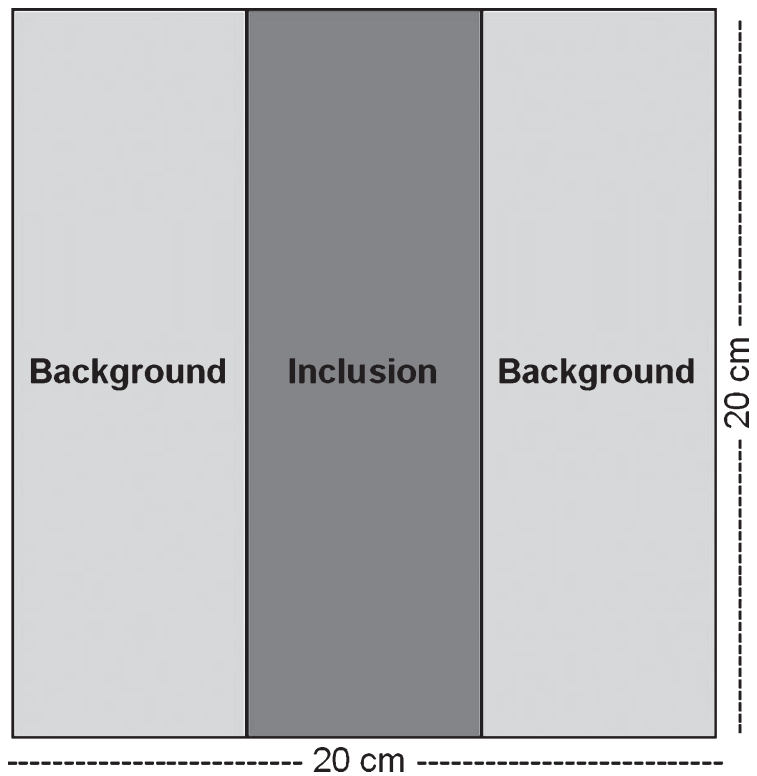

Fig. 13. The protocol of the psychophysical test performed in this work foresees that the user is asked to identify two region of the virtual model, one stiffer (the inclusion) and one softer (the background). The model was implemented using the plain strain approximation, and the model dimension was $20 \mathrm{~cm}$ by $20 \mathrm{~cm}$.

ronment. The subject was asked to interact with the virtual environment by means of the haptic master. The protocol method used for the psychophysical experiments was the method of constant stimuli (MCS), a commonly accepted psychophysical test [13]. Random combinations of stiffness for the inclusion and the background were presented to the subject (Fig. 13), who was asked whether or not he or she could identify that the inclusion was harder than the background. Two sets of experiments were conducted. In the first session, "perfect stiffness" was presented to the subject. "perfect stiffness" means that stiffness is only dependent on the location touched on the surface. The force returned to the operator was the product of the model indentation and the stiffness assigned to that coordinate. The values of stiffness used in this experiment are reported in Table 1, they represent some values in the range of the Young modulus for sound and diseased liver as reported in [36]. The second set of experiments considered the influence of modelling the virtual environment with an FE method, and the "Kalman filter" stiffness estimation. The conditions in this last test are expected to be more noisy with respect to the "perfect stiffness" case, firstly because the FEM has different stiffnesses depending on whether it is tested at the bor-
Table 1

Different values of stiffness used in the experiments, they represent some values in the range of the Young modulus for sound and diseased liver as reported in [36]

\begin{tabular}{lc}
\hline Background $[\mathrm{N} / \mathrm{m}]$ & Inclusion $[\mathrm{N} / \mathrm{m}]$ \\
\hline 25 & 25 \\
25 & 27.5 \\
25 & 30 \\
25 & 32.5 \\
25 & 35 \\
25 & 37.5 \\
\hline
\end{tabular}

der or in the centre (even when the Young modulus is the same for the background and the inclusion), and secondly because the Kalman filter introduces a delay in the stiffness perception. For each session a group of five healthy subjects was considered: one woman and four men with an average age of $30( \pm 5)$ years.

\subsubsection{Data processing}

Aim of the data processing was to extract the threshold for the recognition of two different stiffnesses, this threshold can then be used as evaluation of the system's performances. For each pair of values of background stiffness and inclusion stiffness, was extracted the probability of identification of the harder inclusion $\left(p_{c a}\right)$. It was calculated as the number of correct answers $(\mathrm{ca})$ over the total amount of trials and subjects (sum of $c a$ and non correct answers $(n c a)$ ) as explained by the Eq. 7 .

$$
p_{c a}=\frac{c a}{n c a+c a}
$$

The psychometric function was extracted, fitting the results with a Weibull distribution (8).

$$
p(s)=1-2^{-\left(\frac{s}{\alpha}\right)^{\beta}}
$$

where $\alpha$ and $\beta$ are the parameters characterizing the Weibull distribution, $\alpha$ represents the coherence supporting threshold performance, and $\beta$ is the slope of the curve [16]. The range was fixed between $(0,1)$, the possible answers were fixed at $2, s$ is the stimulus amplitude (measured as absolute stiffness), and $p$ is the probability of identifying the difference. The experimental probability distribution was used to fit the a Weibull distribution. A nonlinear optimization method (the Nelder-Mead method) was used for fitting [17]. The Weibull distribution of $p(0.75)$ was used 
to define difference threshold. The Difference Threshold is the minimum amount of stimulus intensity that produces a noticeable variation in sensory experience. The following relation, known since as Weber's Law, defines the Weber's fraction $(W f)($ Eq. 9):

$$
W f=\frac{\Delta I}{I}
$$

where $\Delta I$ (in this case $=p(0.75)-I)$ is the variation that should imposed to the stimulus $I$ to be perceived as different, in this case the variation of the stimulus is a difference in stiffness [11].

\section{Results}

Figure 14 presents the real stiffness compared with the stiffness estimated with the Kalman filter, in function of the time. Aim of the measurements is to put in evidence the delay in time introduced by the Kalman estimation. In Fig. 15 the time required for the FEM calculation and the data communication through the TCP/IP protocol is reported as a function of the FEM size measured in degrees of freedom (d.o.f.). In Fig. 16 the relative variation of the perceived stiffness when the boundary conditions are varied is reported. The data obtained collected from all the experiments performed on the five subjects were analyzed together. The two Tables 2 and 3 show the collected data, for the perfect stiffness and the Kalman filter respectively.

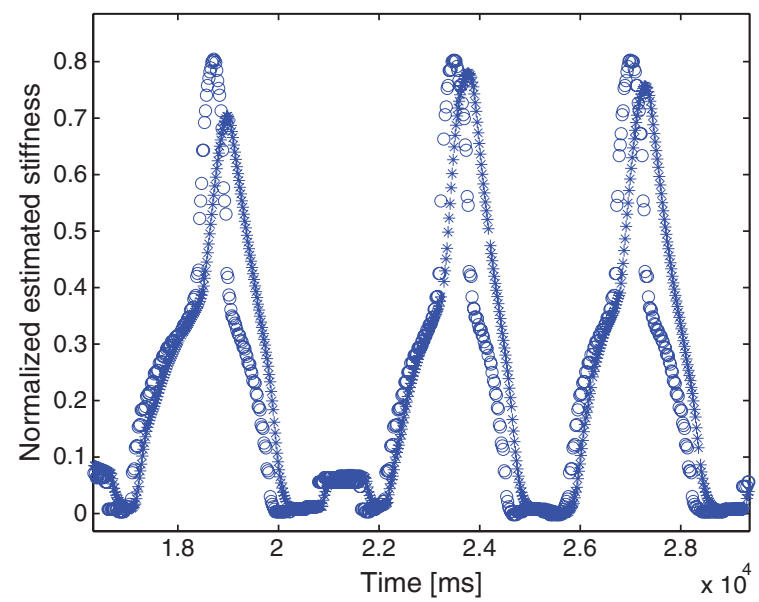

Fig. 14. The model's punctual stiffness has been estimated with a Kalman filter. The figure presents the stiffness estimation vs. time. Real stiffness $\bigcirc$, Kalman filter estimated stiffness *.

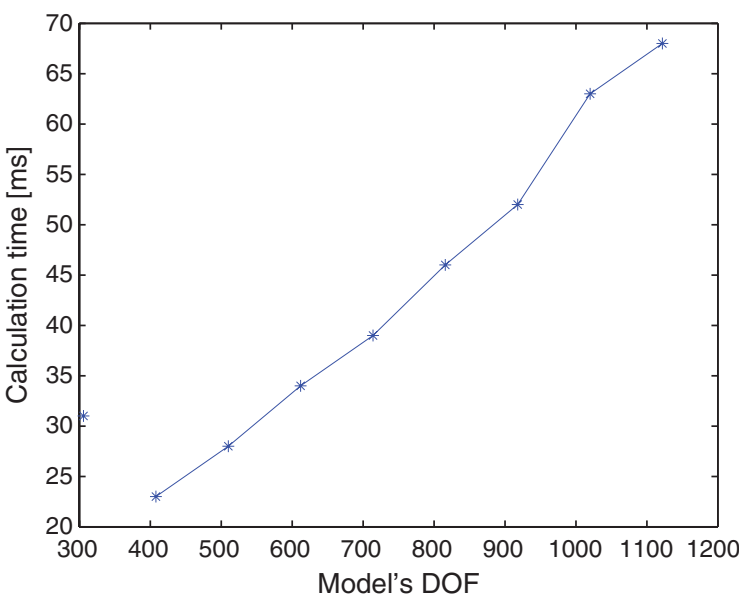

Fig. 15. The amount of degrees of freedom composing the FE model affect strongly the time required for finding the solution. Time required for system solution and result's communication (TCP/IP) in function of the d.o.f. is here presented.

For each experiment are reported the correct answers (ca), meaning that the subject recognized that the two stiffnesses were different, and the non-correct answers (non-ca), meaning that the subject did not recognized the two stiffnesses as different. For each set of data is reported the percentage of correct answers (pca). The $p_{c a}$ was used to fit the psychometric function that is reported in the two figures Figs 17 and 18 for the experiment of the perfect stiffness and Kalman filter

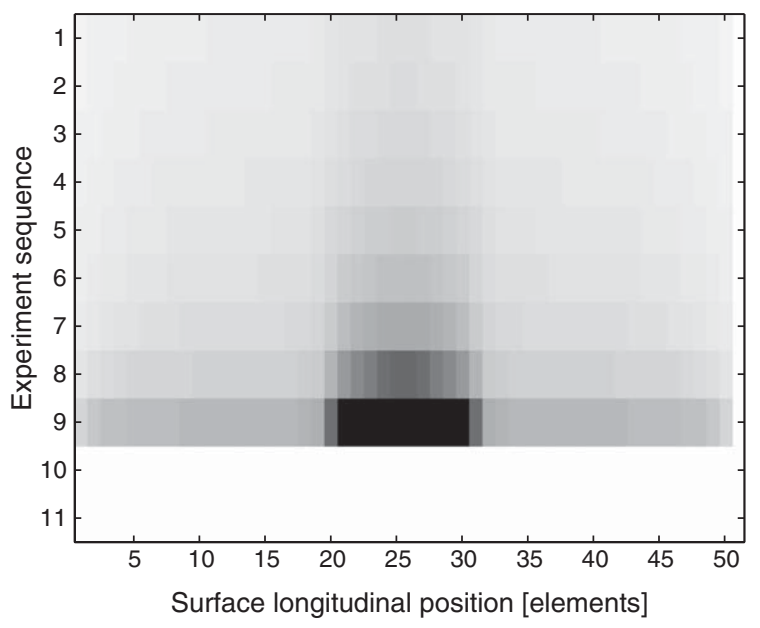

Fig. 16. The perceived stiffness was measured allowing the subject to interact on different surfaces. The figure represents the stiffness along the surface in the nine experiments conducted. The perceived stiffness was normalized over the biggest recorded stiffness. 
Table 2

Data perfect stiffness. The amount of correct answers (ca) non correct answers (nca) and percentage of correct answers are reported in the table. The data were collected using the method of constant stimuli testing the system in the "perfect stiffness" modality

\begin{tabular}{lccc}
\hline Stimulus & $c a$ & $n c a$ & $p_{c a}$ \\
\hline $55(25)$ & 2 & 8 & 0.2 \\
$60(30)$ & 3 & 6 & 0.33 \\
$65(32.5)$ & 2 & 3 & 0.4 \\
$70(35)$ & 5 & 2 & 0.713 \\
$75(37.5)$ & 5 & 0 & 1 \\
\hline
\end{tabular}

Table 3

Data Kalman filter. The amount of correct answers (ca) non correct answers (nca) and percentage of correct answers are reported in the table. The data were collected using the method of constant stimuli testing the system in the "Kalman filter" modality

\begin{tabular}{lccr}
\hline Stimulus & $c a$ & $n c a$ & $p_{c a}$ \\
\hline $55(25)$ & 3 & 2 & 0.6 \\
$60(30)$ & 3 & 2 & 0.6 \\
$65(32.5)$ & 4 & 1 & 0.8 \\
$70(35)$ & 5 & 0 & 1 \\
$75(37.5)$ & 5 & 0 & 1 \\
\hline
\end{tabular}

respectively. by fitting the psychometric function are listed in Table 4 for the "perfect stiffness" and for the "Kalman filter" experimental sessions. The parameters reported are:

I the two values for the Weibull distribution $\alpha$ and $\beta$, and

II the Weber fraction (Wf) calculated using the Ratio value for $p(0.75)$.

\section{Discussion}

This work presented a novel scheme for a teletactile feedback system for minimally invasive surgery. The tactile information is estimated in a quantitative manner using a method based on quantitative elastography. This system is able to implement one of the different algorithms that have been developed for the on-line quantitative estimation of the stiffness based on ultrasound images. The system is designed to be used in combination with a robotic device for minimally invasive surgery. The ultrasound probe is manipulated by the end effector of the robotic arm. The acquired ultra

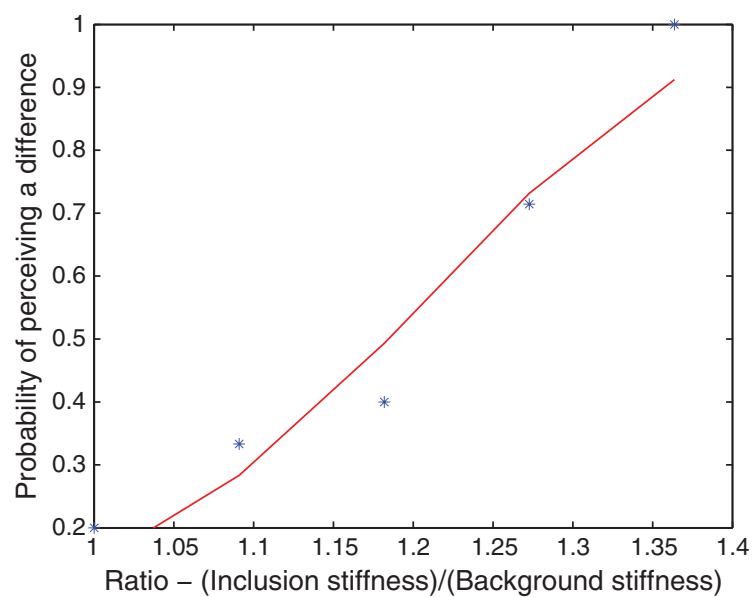

Fig. 17. Psychometric function estimated on the test with the perfect stiffness experiments. The $*$ are the experimental data, the line is the fitted Weibull distribution.

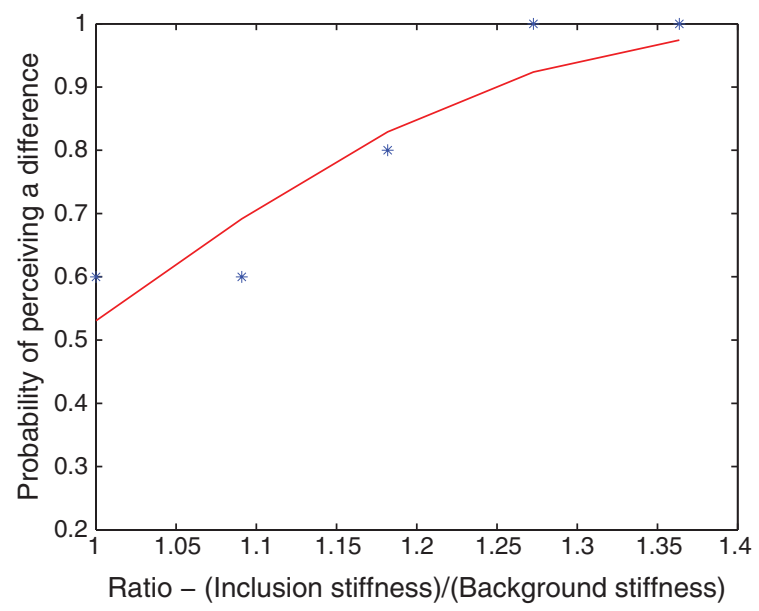

Fig. 18. Psychometric function estimated on the test with the Kalman filter stiffness estimation. The $*$ are the experimental data, the line is the fitted Weibull distribution.

sound images are used for the estimation of the tissue stiffness distribution. An on-line updated virtual environment realizes the interface between the calculated stiffness distribution and the haptic interface used to render the tactile sensation to the user. This approach provides several advantages. Among others was shown how the stiffness perception could be augmented variating the surface on which the model is explored. Figure 16 explains this concept. The identification of hard inclusion in a soft background consists in the identification of the stiffness difference between 
Table 4

Results obtained in the "Perfect stiffness" and "Kalman Filter" experiments

\begin{tabular}{lccc}
\hline & $a$ & $b$ & Wf \\
\hline Perfect stiffness & 32.57 & 8.91 & 0.40 \\
Kalman filter & 27.03 & 5.08 & 0.23 \\
\hline
\end{tabular}

one are respect to another. The closer the touching surface is respect to the harder inclusion the bigger is the contrast between the background stiffness and the inclusion stiffness. This feature brings great advantage because represents a possible way to improve the tactile feedback during the surgery.

Second aspect that was examined is the calculation time required for the solution of the FE problem, this time represents a huge limiting factor for the updating rate of the whole system. The time increases with the increasing of the model's complexity, i.e. with the increasing of the degrees of freedom. Future implementation should evaluate parallel computing solutions.

The update rate affects directly the system's stability, as explained reporting Colgate's work $[6,7]$. The strategy proposed to overcome this problem is based on the physical decoupling of the real time finite element model and the controller of the haptic device, this decoupling is realized introducing a stiffness estimator based on the Kalman filter.

As shown in Fig. 4, the Kalman filter estimation deforms the shape of the stiffness used to control the haptic device, represented mainly by a delay in the correct stiffness representation. The experimental question is then whether or not this estimation could affect the stiffness perceived by the subject. In order to give an answer to this question a set of psychophysical experiments have been performed with the aim of defining a psychometric curve able of quantifying the user's perception.

The psychophysical test has shown that the inclusion's stiffness could be identified in both the "perfect stiffness" (PS) and "Kalman filter" (KF) experiments. The Weber fraction defines the difference in stimulus amplitude that lets the subject discriminate whether the two stimuli are different. The Wf value was found to be bigger for the PS experiments; this value is unexpected because the KF experiments are more noisy and this should affect the discrimination ability. We can assume that such a difference could be due to the variability of the selected population and the two values are comparable. In general the system could provide an understandable difference between the environment and a simulated embedded tumour. Future experiments should extend the examined population in order to reduce the result's variability.

As result of the stiffness estimation performance, it can be observed that the Kalman filter is able to quickly follow the real stiffness; from the experiments only a short delay was recorded. The delay has an effect on the feedback provided to the operator and indeed was reported as an annoying disturbance by many subjects. However the results have shown that a different stiffness due to an harder inclusion could be successfully identified.

\section{Conclusion}

In this work the importance of the tactile feedback in minimally invasive surgery has been discussed. This kind of feedback is of extreme importance in surgical procedures consisting in an essential tool for the diagnosis, as it gives intuitive and immediate information. The case study of procedures for resection of liver metastases has been reported and has shown how is important to locate a hard spot in the liver by means of palpatory procedures.

A teletactile feedback based on Young's modulus estimation, on one hand, enables the use of interesting features like the possibility of touching under the natural surface. On the other hand, it introduces some limitation when integrated in a closed loop system. The limitation studied here is due to the time required to solve the system used to calculate the feedback. This system, implemented as finite element model, requires long time for the calculation, and this time increase with the increasing of the model's degree of freedom (see Fig. 15). The long sampling time can introduce instability in the haptic interface control loop, as explained in Sec. 3.4.1. This drawback could be compensated with the introduction of a stiffness estimation block implemented as extended Kalman filter. The psychophysical experiment conducted in this work demonstrated that although the filter introduces a phase lag in the estimation (see Fig. 14), this does not hinder the user from perceiving the correct stiffness, as results for the calculated Wf (see Table 4). Future works will study the implementation of a faster solver for the FE model and the testing of the system in a mock up of the surgical environment. 


\section{References}

[1] J. Alberty, C. Carstensen, S.A. Fiunken and R. Klose, Matlab implementation of the finite element method in elasticity, Computing 69(3) (November 2002), 239-263.

[2] K.E. Atkinson, An Introduction to Numerical Analysis, John Wiley \& Sons, New York, 1989.

[3] P.E. Barbone and N.H. Gokale, Elastic modulus imaging: on the uniqueness and nonuniqueness of the elastography inverse problem in two dimensions, Inverse Problems 20 (2004), 283-296.

[4] J. Berkley, G. Turkiyyah, D. Berg, M. Ganter and S. Weghorst, Real-time finite element modeling for surgery simulation: An application to virtual suturing, IEEE Transactions on Visualization and Computer Graphics 10(3) (May 2004), 314325

[5] J. Bercoff, M. Tanter and M. Fink, Supersonic shear imaging: A new technique for soft tissue elasticity mapping, IEEE Trans Ultrason Ferroelectr Freq Control 51(4) (April 2004), 396-409.

[6] J.E. Colgate and J.M. Brown, Factors affecting the Z-width of a haptic display, in: Proc IEEE International Conference on Robotics and Automation, May 1994, pp. 3205-3210.

[7] J.E. Colgate and G. Schenkel, Passivity of a class of sampleddata systems: Application to haptic interfaces, in: Proc American Control Conference, Vol. 3, June 1994, pp. 32363240 .

[8] J. Camino, M. Sette and M. Diehl, Young's modulus reconstruction using a constrained Gauss-Newton method, in: Book of Abstracts of 26th Benelux Meeting on Systems and Control, Lommel, Mar. 2006.

[9] M.M. Doyley, J.C. Bamber, F. Fuechsel and N.L. Bush, A freehand elastographic imaging approach for clinical breast imaging: System development and performance evaluation, Ultrasound Med Biol 27(10) (October 2001), 1347-1357.

[10] G. De Gersem, Kinaesthetic Feedback and Enanced Sensitivity in Robotic Endoscopic Telesurgery, $\mathrm{PhD}$ thesis, Katholieke Universiteit Leuven, Faculty Mechanical Engineering, 2005.

[11] G. Deco, L. Scarano and S. Soto-Faraco, Weber's law in decision making: integrating behavioral data in humans with a neurophysiological model, The Journal of Neuroscience : The Official Journal of the Society for Neuroscience 27(42) (October 2007), 11192-1200.

[12] J. W. Eaton, GNU Octave Manual, Network Theory Limited, 2002

[13] G. Gescheider, The Classical Psychophysical Methods, Psychophysics: The Fundamentals, chapter 3, Lawrence Erlbaum Associates, 1997.

[14] J. Leven, D. Burschka, R. Kumar, G. Zhang, S. Blumenkranz, X. Donald Dai, M. Awad, G.D. Hager, M. Marohn, M. Choti, C. Hasser and R.H. Taylor, DaVinci canvas: A telerobotic surgical system with integrated, robot-assisted, laparoscopic ultrasound capability, Med Image Comput Comput Assist Interv Int Conf Med Image Comput Comput Assist Interv $\mathbf{8}(\mathrm{Pt}$ 1) (2005), 811-818.

[15] M.H. Lee and H.R. Nicholls, Review article tactile sensing for mechatronics - a state of the art survey, Mechatronics 9(1) (1999), 1-31.

[16] J.R. Müller, M.G. Philiastides and W.T. Newsome, Microstimulation of the superior colliculus focuses attention without moving the eyes, Proceedings of the National Academy of Sciences of the United States of America 102(3) (2005), 524-549.
[17] J.A. Nelder and R. Meadf, A simplex method for function minimization, The Computer Journal 7(4) (1964), 308-313.

[18] J. Nocedal and S.J. Wright, Numerical Optimization, Springer, August 1999.

[19] J. Ophir, K. Alam, B. Garra, F. Kallel, E. Konofagou and T. Varghese, Elastography: Imaging of tissue elastic properties in vivo, in: Proc IEEE Ultrasonics Symposium, Vol. 2, October 1998, p. 1743.

[20] J. Ophir, S.K. Alam, B. Garra, F. Kallel, E. Konofagou, T. Krouskop and T. Varghese, Elastography: Ultrasonic estimation and imaging of the elastic properties of tissues, Proc Inst Mech Eng H 213(3) (1999), 203-233.

[21] | J. Ophir, S.K. Alam, B.S. Garra, F. Kallel, E.E. Konofagou, T. Krouskop, C.R.B Merrit, R. Righetti, R. Souchon, S. Srinivasan and T. Varghese, Elastography: Imaging the elastic properties of soft tissues with ultrasound, J Med Ultrasonics 29 (2002), 155-171.

[22] J. Ophir, I. Cespedes, H. Ponnekanti, Y. Yazdi and X. Li, Elastography: A quantitative method for imaging the elasticity of biological tissues, Ultrason Imaging 13(2) (April 1991), $111-134$.

[23] J. Ophir, B. Garra, F. Kallel, E. Konofagou, T. Krouskop, R. Righetti and T. Varghese, Elastographic imaging, Ultrasound Med Biol 26(Suppl 1) (May 2000), S23-S29.

[24] W.J. Peine, P.S. Welleman and R.D. Howe, Temporal bandwidth requirements for tactile shape displays, in: Sith Annual Symposium on Haptic Interfaces for Virtual Environment and Teleoperator Systems, ASME International Mechanical Engineering Congress and Expositions, 1997.

[25] K. Salisbury, F. Conti and F. Barbagli, Haptic rendering: Introductory concepts, IEEE Computer Graphics and Applications 24(2) (March 2004), 24-32.

[26] M. Sette, J. Camino, J. D'hooge, H. Van Brussel and J. Vander Sloten, Comparing optimization algorithms for Young's modulus reconstruction In. ultrasound elastography, In 2007 IEEE Ultrasonics Symposium Proceedings, 2007.

[27] M.M. Sette, J.F. Camino, J. D’hooge, H. Van Brussel and J. Vander Sloten, P4F-3 comparing optimization algorithms for the young's modulus reconstruction in ultrasound elastography, in: Proc IEEE Ultrasonics Symposium, October 2007, pp. 2028-2031.

[28] M. Sette, F. Conversano, H. Van Brussel and J. Vander Sloten, Minimally Invasive Technologies and Nanosystems for Diagnosis and Therapies, chapter Elastotouc, Lupiensis Biomedical Publications, 2008, pp. 43-50.

[29] M. Sette, J. D'hooge, S. Langeland, P. Goethals, H. Van Brussel and J. Vander Sloten, Tactile feedback in minimally invasive procedures using an elastography-based method, Int J CARS S504, 2007.

[30] M. Sette, Technological Contributions to Augmented Reality in Minimal Invasive Surgery, PhD thesis, Katholieke Univesiteit Leuven, Leuven, 2010, p. 240. doi: D/2010/7515/81

[31] S. Schostek, M.O Schurr and G.F. Buess, Medical engineering \& physics review on aspects of artificial tactile feedback in laparoscopic surgery, Medical Engineering \& Physics 31 (2009), 887-898.

[32] L. Sandrin, M. Tanter, S. Catheline and M. Fink, Shear modulus imaging with 2-D transient elastography, IEEE Transactions on Ultrasonics, Ferroelectrics, and Frequency Control 49(4) (April 2002), 426-435.

[33] W. Richard Stevens, TCP/IP Illustrated, Vol. I: The Protocols, Addison-Wesley, Reading, MA, 1994. 
[34] M. Sette, J. Vander Sloten and H. Van Brussel, New Technology Frontiers in Minimally Invasive Therapies, chapter Estimation, Lupiensis Biomedical Publications, 2007, pp. 80-88.

[35] A.L. Trejos, J. Jayender, M.T. Perri, M.D. Naish, R.V. Patel and R.A. Malthaner, Robot-assisted tactile sensing for mini- mally invasive tumor localization, Int J Rob Res 28(9) (2009) 1118-1133.

[36] W.-C. Yeh, Y.-M. Hsu, P.-L. Kuo, M.-L. Li, P.-M. Yang, P.-H Lee and P.-C. Li, IEEE ultrasonics symposium-1234, 2001 IEEE Ultrasonics Symposium, 2001, pp. 1233-1236. 

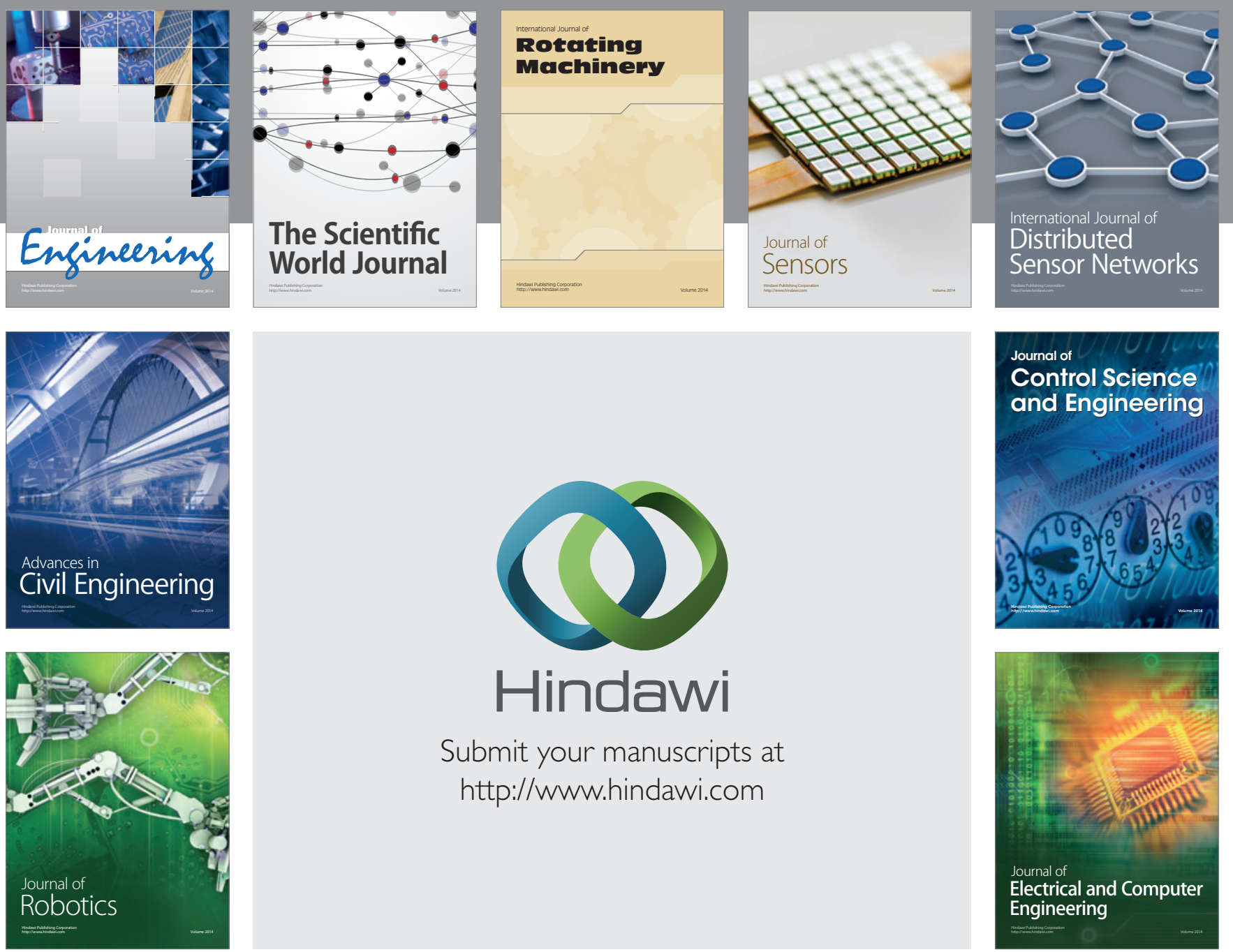

Submit your manuscripts at

http://www.hindawi.com
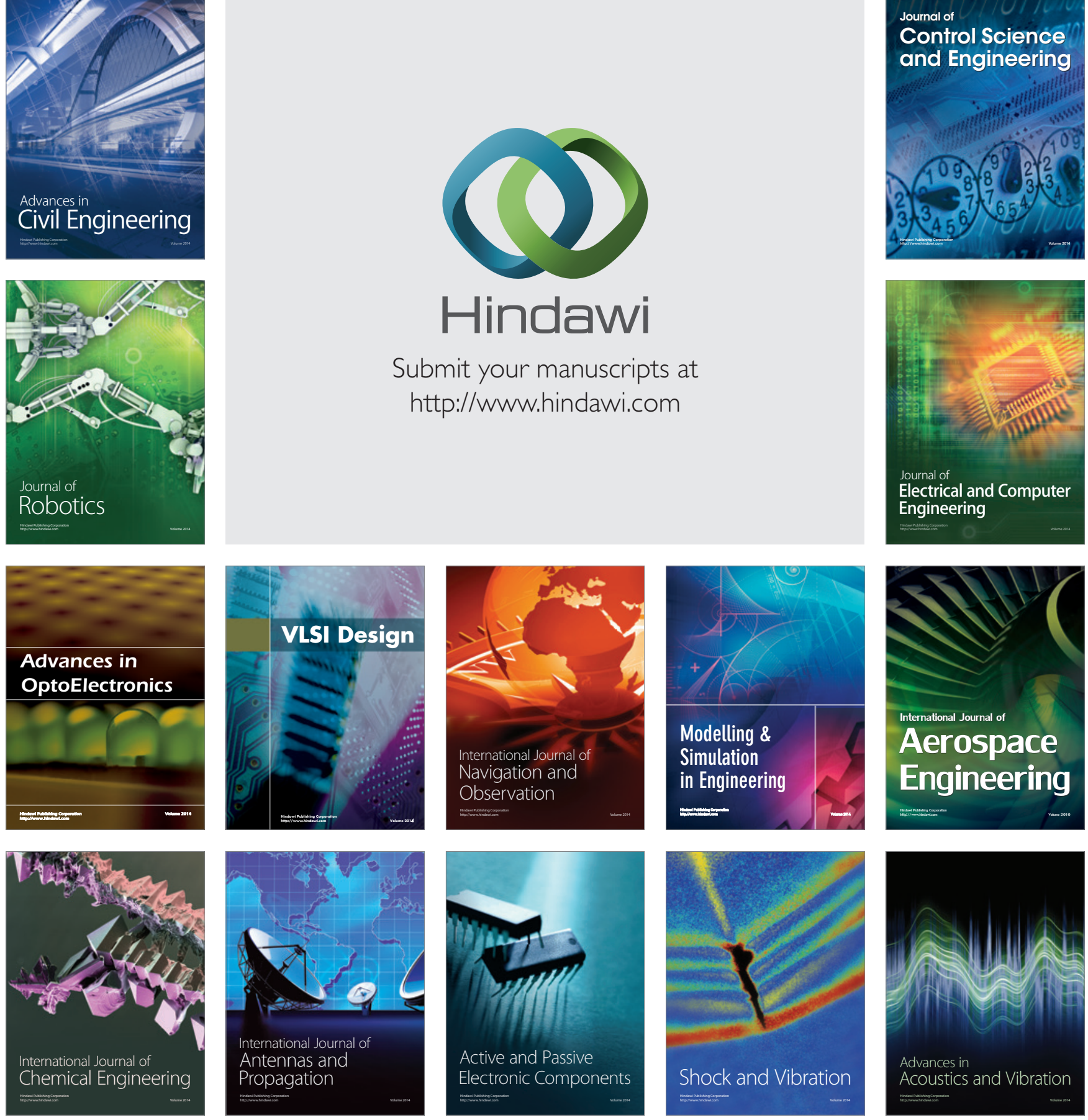